\title{
Evaluating soil metallic pollution and consequent human health hazards in the vicinity of an industrialized zone, case study of Mubarakeh steel complex, Iran
}

\author{
Zohreh Ghaemi ${ }^{1}$, Abdolreza Karbassi ${ }^{2 *}$, Faramarz Moattar ${ }^{1}$, Amirhesam Hassani ${ }^{1}$ and Nematollah Khorasani ${ }^{1}$
}

\begin{abstract}
Background: Being established in 1988 in the vicinity of Isfahan city, Mubarakeh Steel complex has imposed adverse environmental and health effects within the area. The study area is covered by lots of farms through which major crops like wheat and rice are provided.

Methods: Considering the imposed pollution load of the complex, the current study has monitored the concentration of metals $\mathrm{Fe}, \mathrm{Al}, \mathrm{Cd}, \mathrm{Cr}, \mathrm{Ni}, \mathrm{Pb}, \mathrm{Cu}, \mathrm{Zn}, \mathrm{Mn}, \mathrm{Co}, \mathrm{Mo}$, As in 14 soil samples within the study area. Furthermore, human health hazards of mentioned metals due to consumption of domestic rice and wheat have also been evaluated through different scenarios. In order to evaluate the mobility of metals in soil samples the sequential chemical analysis is performed.

Results: Regarding the accumulation of metals in loose phases the order of metals bioavailability risk level is estimated to be as follows:

$\mathrm{Co}>\mathrm{Cd}>\mathrm{Mo}>\mathrm{Ba}>\mathrm{As}>\mathrm{Pb}>\mathrm{Mn}>\mathrm{Cu}>\mathrm{V}>\mathrm{Zn}>\mathrm{Cr}>\mathrm{Ni}$

Discussion: An index approach is also considered to evaluate the severity of metal contamination. Regarding geochemical accumulation index, only cadmium is detected to be in a moderately contaminated status while other metals declare an unpolluted condition. Index of pollution pays more attention to mobility potential of metals and accordingly detects metals $\mathrm{Co}, \mathrm{Mn}, \mathrm{As}, \mathrm{Pb}, \mathrm{Cd}, \mathrm{Ba}$ and $\mathrm{Mo}$ to be in a moderately contaminated level. On the other hand, enrichment factor declares all toxic metals except for $\mathrm{Co}, \mathrm{Ba}$ and $\mathrm{V}$ to be enriched.
\end{abstract}

Conclusions: Considering human health hazard assessment, except for $\mathrm{Fe}, \mathrm{Ba}$, $\mathrm{Cu}$ and $\mathrm{Zn}$, all metals intakes in different scenarios are considered as hazardous while their $C D I$ values are much more than the respective oral reference doses.

Keywords: Metal pollution, Soil, Mobility, Mubarakeh steel complex, Isfahan

\section{Introduction}

Metal contaminated sites contain a potentially hazardous risk for human beings and the environment. The bioavailability of such pollution is sophisticatedly used as an indicator of potential risk $[1,2]$. Metals may exist in various species in the environment, where they may be transformed from one form into another or exist in different forms simultaneously. Depending on environmental conditions, the chemical speciation of metals changes widely. Such differences in chemical

\footnotetext{
*Correspondence: akarbasi@ut.ac.ir

${ }^{2}$ Graduate Faculty of Environment, University of Tehran, P.O. Box 14155-6135 Tehran, Iran

Full list of author information is available at the end of the article
}

speciation play a key role in forming the environmental fate, bioaccessibility and environmental risk of the metals.

Knowledge on metals bulk concentrations alone may not be sufficient enough to evaluate the adverse effects of contaminated soils while toxic metals are present in different chemical forms in soil (easily exchangeable ions, metal carbonates, oxides, sulfides, organometallic compounds, ions in crystal lattices of minerals, etc.), which determine their mobilization capacity and bioavailability [3-5].

Knowledge of metal's speciation may help to evaluate the metal tendency to remain in soil or sediments and how easily it may be released into the water phase.

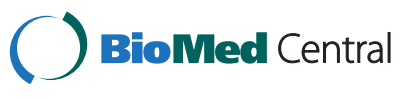

(c) 2015 Ghaemi et al. Open Access This article is distributed under the terms of the Creative Commons Attribution 4.0 International License (http://creativecommons.org/licenses/by/4.0/), which permits unrestricted use, distribution, and reproduction in any medium, provided you give appropriate credit to the original author(s) and the source, provide a link to the Creative Commons license, and indicate if changes were made. The Creative Commons Public Domain Dedication waiver (http://creativecommons.org/publicdomain/zero/1.0/) applies to the data made available in this article, unless otherwise stated. 
Sequential extraction procedures are among the most widely used methods through which several extractants are used consecutively to separate the portion of phases from the bulk metallic concentration in a soil sample.

A vast variety of extraction processes providing information about the strength of bonds between metal species and soil fractions have been used by different researchers [2, 6-8]. However, none has been fully accepted as a universal method to be applied for all types of soils metallic pollution studies. Such lack of integrity may result in an erroneous diagnosis in soils. The study of potential mobility involves summing up all reactions that are capable of mobilising metals [9].

Being established in 1988 in the vicinity of Mubarakeh county, Mubarakeh Steel complex has imposed adverse environmental and health effects within the area. A remarkable increase in the number of reported disease cases in Mubarakeh and Zarrinshahr in recent years may be attributed to the mentioned complex activities. The study area is covered by lots of farms through which major crops of Isfahan province are provided. The study area within Iran is demonstrated in Fig. 1.

Considering the imposed pollution load of the complex, the current study has monitored the concentration of metals iron, aluminum, cadmium, chromium, nickel, lead, copper, zinc, manganese, cobalt, molybdenum and arsenic in soil samples within the study area.

The current study presents not only the bulk concentration of eight metals ( $\mathrm{Fe}, \mathrm{Al}, \mathrm{Cd}, \mathrm{Cr}, \mathrm{Ni}, \mathrm{Pb}, \mathrm{Cu}, \mathrm{Zn}, \mathrm{Mn}$,
Co, Mo, As) in superficial soil samples of Mubarakeh steel complex vicinity, but also the chemical partitioning of mentioned elements. The latter findings create a clear view of metals potential mobility and consequently the tendency to be absorbed by plants or animals within the bioaccessibility phenomenon. For this study, a modified sequential extraction procedure has been considered [10, 11].

\section{Materials and methods}

Soil samples were collected from 14 stations in the vicinity of Mubarakeh steel complex during the summer of 2012 using a Peterson grab sampler (Fig. 2). Spatial details of sampling locations are indicated in Table 1.

Soil samples, which were brown to grey in color, were air-dried and passed through a 63- $\mu \mathrm{m}$ mesh (equivalent to a No. 230 sieve, ASTM E-11). Being powdered using an agate mortar and pestle, about $0.5 \mathrm{~g}$ of the sample was placed in a Teflon beaker containing $10 \mathrm{~mL}$ aqua regia.

The mixture was heated until most of the liquid had evaporated, and allowed to cool before $5 \mathrm{~mL}$ of hydrogen fluoride (HF) were added. The samples were further cooled to room temperature before being filtered. The filtrates were transferred to $50 \mathrm{~mL}$ volumetric flasks and brought to volume with $1 \mathrm{~N} \mathrm{HCl}$ [10]. Slightly modified from the old procedure, chemical partitioning was conducted in three sequential steps: (1) acetic acid $25 \% \mathrm{v} / \mathrm{v}$,

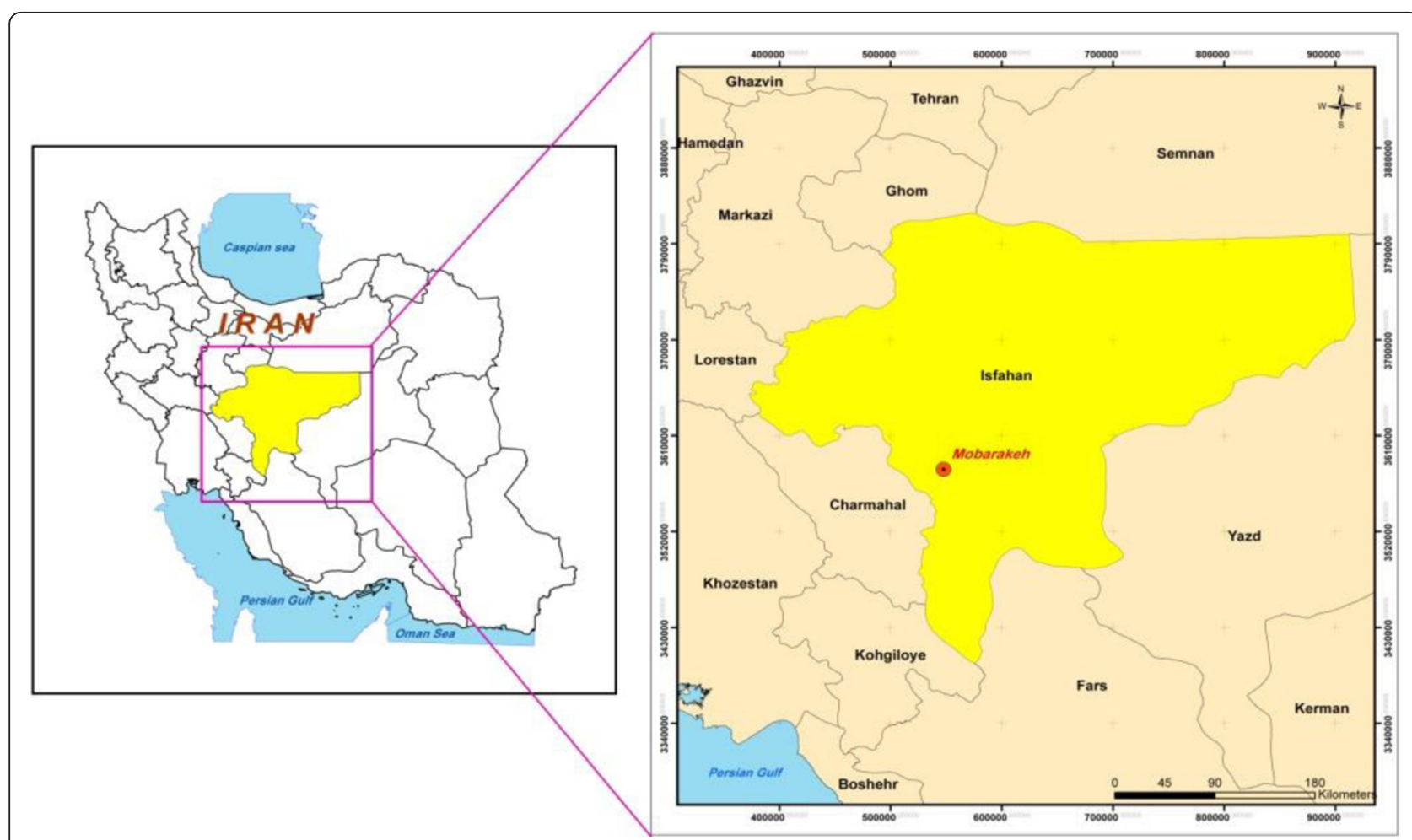

Fig. 1 Study area within Iran and Isfahan province 


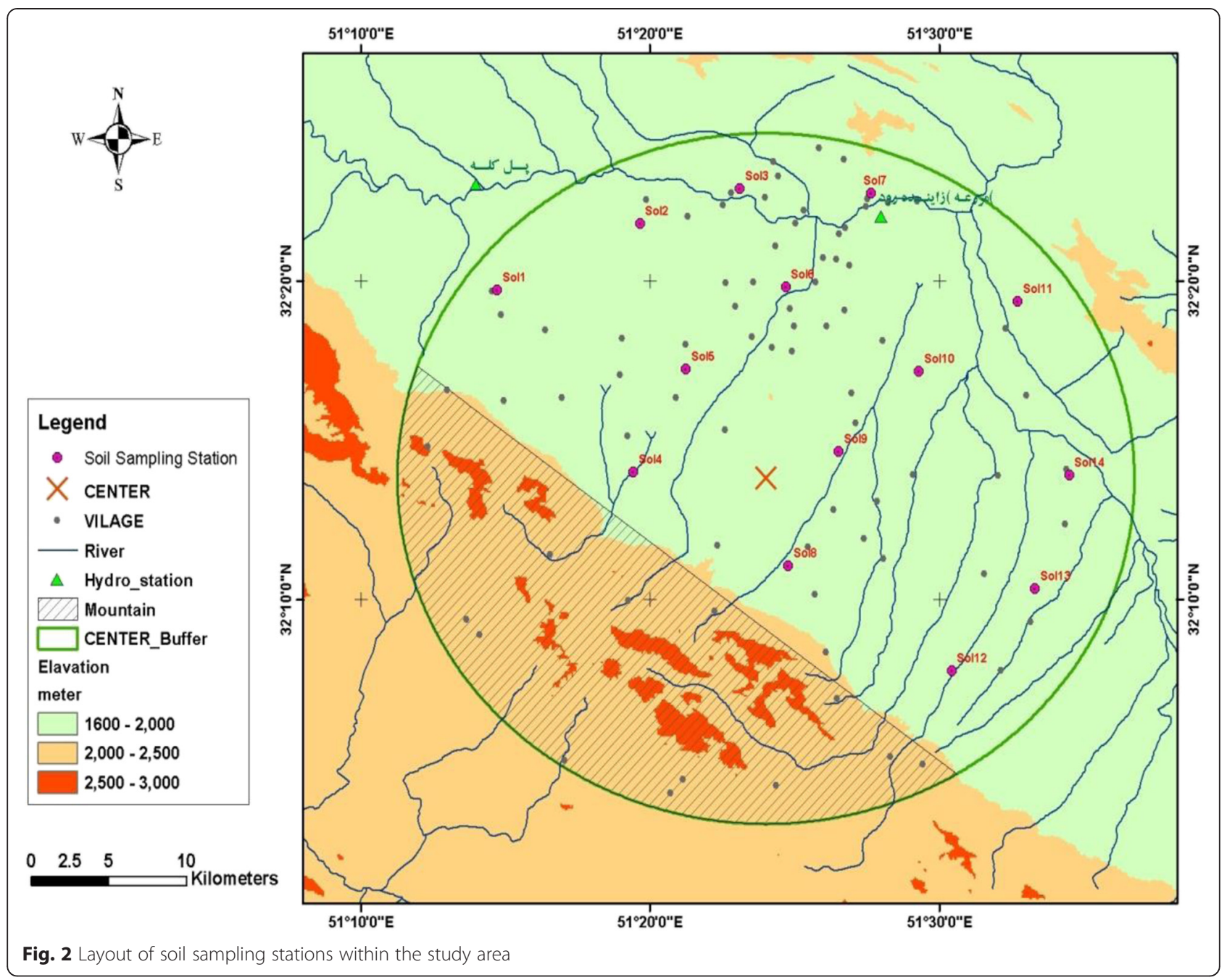

Table 1 Spatial characteristics of soil sampling stations

\begin{tabular}{lll}
\hline Station & UTM X & UTM Y \\
\hline 1 & 51.24535 & 32.32831 \\
2 & 51.32745 & 32.36294 \\
3 & 51.38493 & 32.3814 \\
4 & 51.32361 & 32.23317 \\
5 & 51.35387 & 32.28718 \\
6 & 51.41157 & 32.33025 \\
7 & 51.46045 & 32.37887 \\
8 & 51.4129 & 32.18419 \\
9 & 51.44207 & 32.24389 \\
10 & 51.48821 & 32.28587 \\
11 & 51.54518 & 32.32231 \\
12 & 51.50707 & 32.12949 \\
13 & 51.55509 & 32.17231 \\
14 & 51.57471 & 32.23179 \\
\hline
\end{tabular}

(2) acetic acid $25 \% \mathrm{v} / \mathrm{v}-0.1 \mathrm{M}$ hydroxylamine hydrochloride and (3) $30 \% \mathrm{H} 202$ "extraction with $1 \mathrm{M}$ ammonium acetate" [11]. To digest rice and wheat samples, 15 milliliters of the mixture $\mathrm{HNO}_{3}: \mathrm{H}_{2} \mathrm{SO}_{4}: \mathrm{HClO}_{4}$ 1:1:5 was added to 1 gram of powdered rice/wheat and heated to $80^{\circ}$ centigrade. The mixtures were further cooled and filtered before injection process [12].

The analysis of metals in solutions was carried out by an inductively coupled plasma atomic emission spectrometer. Certified reference material (CRM 320) was used to check the accuracy of the analytical procedures. Replicate analysis of this CRM showed an acceptable accuracy, with recovery rates for metals between $92 \%$ and

Table 2 Details of rice consumption scenarios within the study area

\begin{tabular}{lllllll}
\hline Scenario No. & 1 & 2 & 3 & 4 & 5 & 6 \\
\hline Body weight (kg) & 70 & 70 & 70 & 15 & 15 & 15 \\
Daily rice consumption (gr) & 100 & 250 & 400 & 100 & 250 & 400
\end{tabular}


Table 3 Details of wheat consumption scenarios within the study area

\begin{tabular}{lllllll}
\hline Scenario No. & 1 & 2 & 3 & 4 & 5 & 6 \\
\hline Body weight (kg) & 70 & 70 & 70 & 15 & 15 & 15 \\
Daily wheat consumption (gr) & 150 & 300 & 450 & 150 & 300 & 450 \\
\hline
\end{tabular}

$105 \%$. Furthermore, two samples were analyzed in triplicate to determine the precision of the analytical processes. The average values of the variation coefficients were estimated to be less than $10 \%$ which may be considered acceptable for such studies [13].

To have an estimate of background status, according to soil samples collected from the vicinity of Mubarakeh steel, metals concentrations in shale have been considered. Such assumption is normally used as background values in sediment studies [13-16].

In order to determine the value of metal concentrations in different sedimentary phases, the modified sequential chemical partitioning method was considered [11].

To evaluate the severity of metallic pollution within the study area, geochemical accumulation index, enrichment factor and index of pollution were taken into consideration.

An index approach was used to assess the severity of metal contamination in the vicinity of Mubarakeh steel complex. Accordingly, the geochemical accumulation index was calculated using:

$$
\text { Igeo }=\log 2[\mathrm{Cn} /(1.5 * \mathrm{Bn})]
$$

Where Igeo stands for geochemical accumulation index, $\mathrm{Cn}$ is the bulk metal concentration and $\mathrm{Bn}$ is the average metal concentration in shale $[17,18]$.

The enrichment factor (EF) values was calculated for metals using:

$$
\mathrm{EF}=[(\mathrm{Cn} / \mathrm{CFe}) \text { sample }] /[(\mathrm{Cn} / \mathrm{CFe}) \text { crust }]
$$

Where $(\mathrm{Cn} / \mathrm{CFe})$ sample indicates the ratio of the concentration of a specific element $(\mathrm{Cn})$ to that of $\mathrm{Fe}(\mathrm{CFe})$ in soil sample and $(\mathrm{Cn} / \mathrm{CFe})$ crust is the same ratio in an unpolluted reference sample [19].

Finally, index of pollution which is a modification of Igeo is referred by the following formula:

$$
\mathrm{IPOLL}=\log 2[\mathrm{Bc} / \mathrm{Lp}]
$$

The study area is a major agricultural site in the province and crops like wheat and rice are widely cultivated within the region. In order to evaluate the probable crops metal uptake, metals concentration in eight different rice and 15 different wheat samples were measured.

To evaluate the hazard level threatening local habitants due to consumption of rice and wheat products, nutritional pattern and physiological characteristics of residents should be taken into consideration. Accord-

\begin{tabular}{|c|c|c|c|c|c|c|c|c|c|c|c|c|c|c|}
\hline Station & Co & $\mathrm{Mn}$ & As & $\mathrm{Ni}$ & $\mathrm{Pb}$ & $\mathrm{Cr}$ & $\mathrm{Cd}$ & $\mathrm{Cu}$ & $\mathrm{Ba}$ & Mo & $\mathrm{Zn}$ & V & $\mathrm{Fe} \%$ & $\mathrm{Al} \%$ \\
\hline$\overline{S 1}$ & 1.80 & 391.98 & 6.39 & 49.63 & 6.84 & 53.66 & 0.98 & 27.46 & 94.31 & 0.69 & 70.85 & 25.27 & 1.89 & 0.10 \\
\hline S2 & 2.31 & 455.58 & 6.67 & 47.35 & 2.84 & 38.22 & 0.92 & 33.08 & 95.64 & 0.45 & 67.03 & 25.64 & 1.37 & 0.10 \\
\hline S3 & 5.88 & 419.01 & 6.28 & 45.91 & 7.58 & 40.77 & 0.84 & 72.39 & 68.33 & 0.69 & 87.91 & 31.49 & 1.45 & 0.11 \\
\hline S4 & 3.90 & 501.45 & 8.56 & 64.20 & 20.84 & 47.45 & 4.34 & 70.18 & 98.77 & 0.35 & 95.72 & 27.34 & 1.61 & 0.11 \\
\hline S5 & 3.66 & 603.02 & 9.99 & 80.00 & 8.62 & 59.70 & 1.24 & 59.07 & 98.87 & 0.49 & 102.81 & 25.78 & 2.19 & 0.12 \\
\hline S6 & 4.90 & 560.10 & 8.43 & 65.06 & 8.42 & 47.19 & 1.08 & 44.01 & 87.62 & 0.47 & 77.03 & 24.55 & 1.64 & 0.13 \\
\hline S7 & 2.37 & 465.56 & 7.30 & 65.21 & 10.61 & 45.64 & 1.14 & 35.89 & 100.81 & 0.61 & 80.71 & 22.70 & 1.85 & 0.13 \\
\hline S8 & 2.43 & 445.69 & 8.64 & 66.53 & 7.55 & 43.88 & 0.98 & 31.68 & 71.48 & 0.76 & 73.95 & 23.26 & 1.58 & 0.12 \\
\hline S9 & 2.18 & 341.01 & 7.34 & 59.28 & 6.41 & 46.01 & 4.77 & 38.51 & 88.40 & 0.24 & 68.30 & 19.04 & 1.43 & 0.12 \\
\hline $\mathrm{S} 10$ & 3.03 & 421.85 & 7.14 & 59.58 & 7.93 & 42.74 & 1.01 & 29.38 & 73.71 & 0.28 & 70.09 & 18.26 & 1.46 & 0.10 \\
\hline S11 & 1.73 & 414.25 & 8.42 & 58.69 & 10.77 & 42.94 & 1.06 & 36.40 & 93.12 & 0.41 & 87.59 & 21.18 & 1.50 & 0.12 \\
\hline $\mathrm{S} 12$ & 1.88 & 466.20 & 8.88 & 69.48 & 9.55 & 47.47 & 1.10 & 32.02 & 75.65 & 0.72 & 79.21 & 20.92 & 1.67 & 0.13 \\
\hline S13 & 1.00 & 398.80 & 9.01 & 70.65 & 8.11 & 47.77 & 1.07 & 31.34 & 68.14 & 0.64 & 81.15 & 16.91 & 1.70 & 0.13 \\
\hline S14 & 2.04 & 341.36 & 11.20 & 55.94 & 8.49 & 37.43 & 0.91 & 39.63 & 68.66 & 0.31 & 74.13 & 20.28 & 1.34 & 0.11 \\
\hline Max & 5.88 & 603.02 & 11.20 & 80.00 & 20.84 & 59.70 & 4.77 & 72.39 & 100.81 & 0.76 & 102.81 & 31.49 & 2.19 & 0.13 \\
\hline Min & 1.00 & 341.01 & 6.28 & 45.91 & 2.84 & 37.43 & 0.84 & 27.46 & 68.14 & 0.24 & 67.03 & 16.91 & 1.34 & 0.10 \\
\hline SD & 1.35 & 73.93 & 1.41 & 9.52 & 3.94 & 5.82 & 1.29 & 14.85 & 12.83 & 0.18 & 10.58 & 3.96 & 0.23 & 0.01 \\
\hline Mean & 2.79 & 444.70 & 8.16 & 61.25 & 8.90 & 45.78 & 1.53 & 41.50 & 84.54 & 0.51 & 79.75 & 23.04 & 1.62 & 0.12 \\
\hline Shale values & 19 & 850 & 13 & 68 & 20 & 90 & 0.3 & 45 & 498 & 2.6 & 95 & 130 & 4.70 & 8.2 \\
\hline
\end{tabular}
ingly six different scenarios are defined (Tables 2 and 3).

Table 4 Bulk concentration of metals ( $\mathrm{mg} / \mathrm{kg}$, dry mass) in soil samples within the study area 


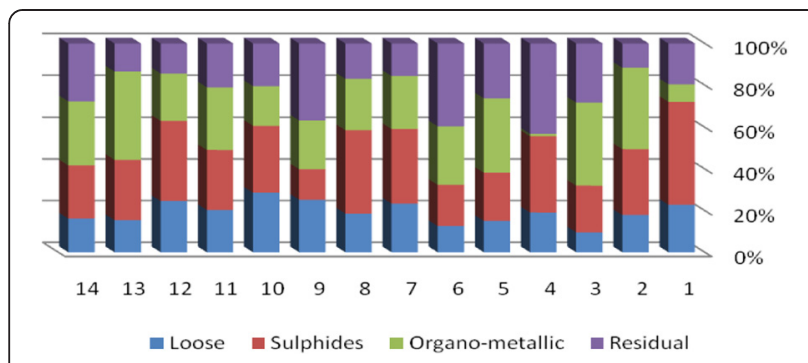

Fig. 3 Chemical partitioning of Cobalt in soil samples

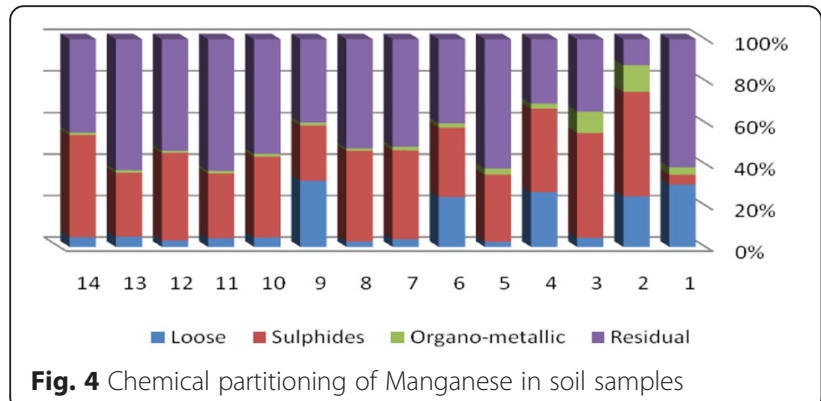

Fig. 4 Chemical partitioning of Manganese in soil samples

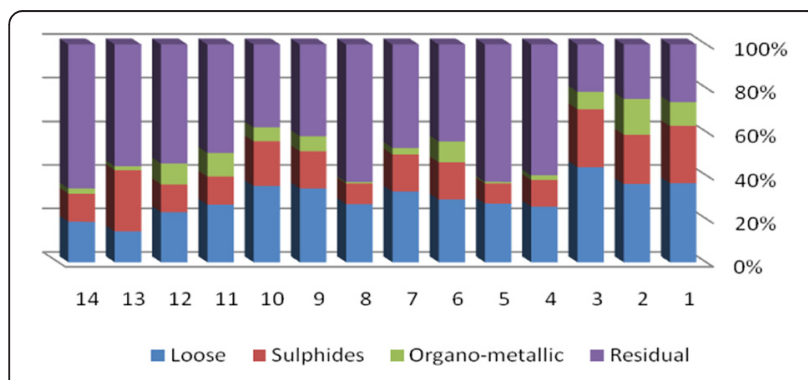

Fig. 5 Chemical partitioning of Arsenic in soil samples
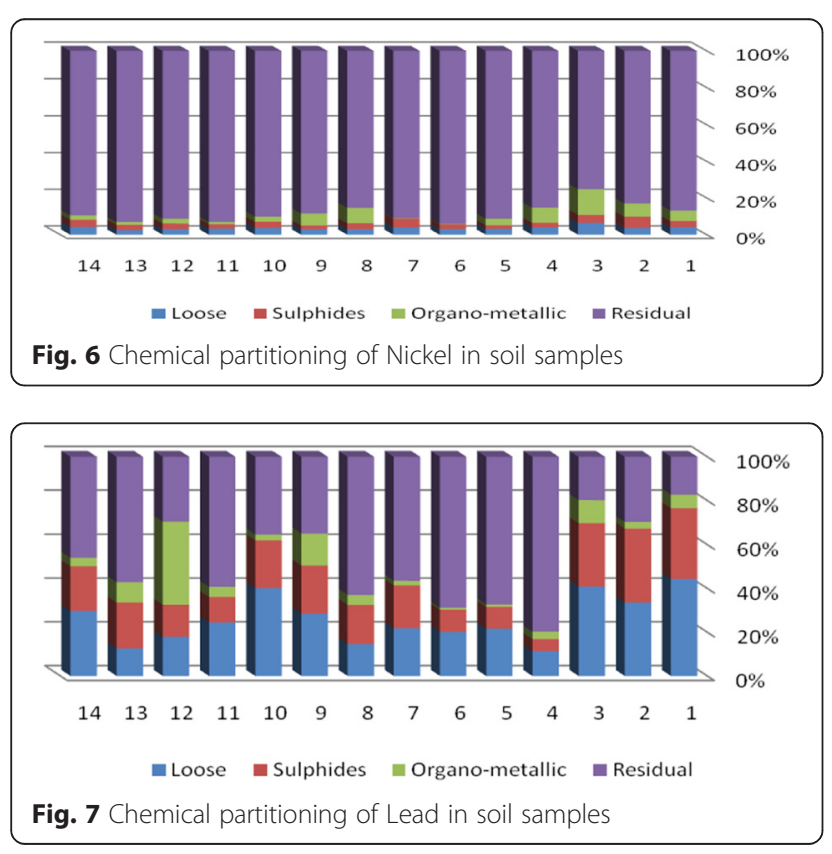
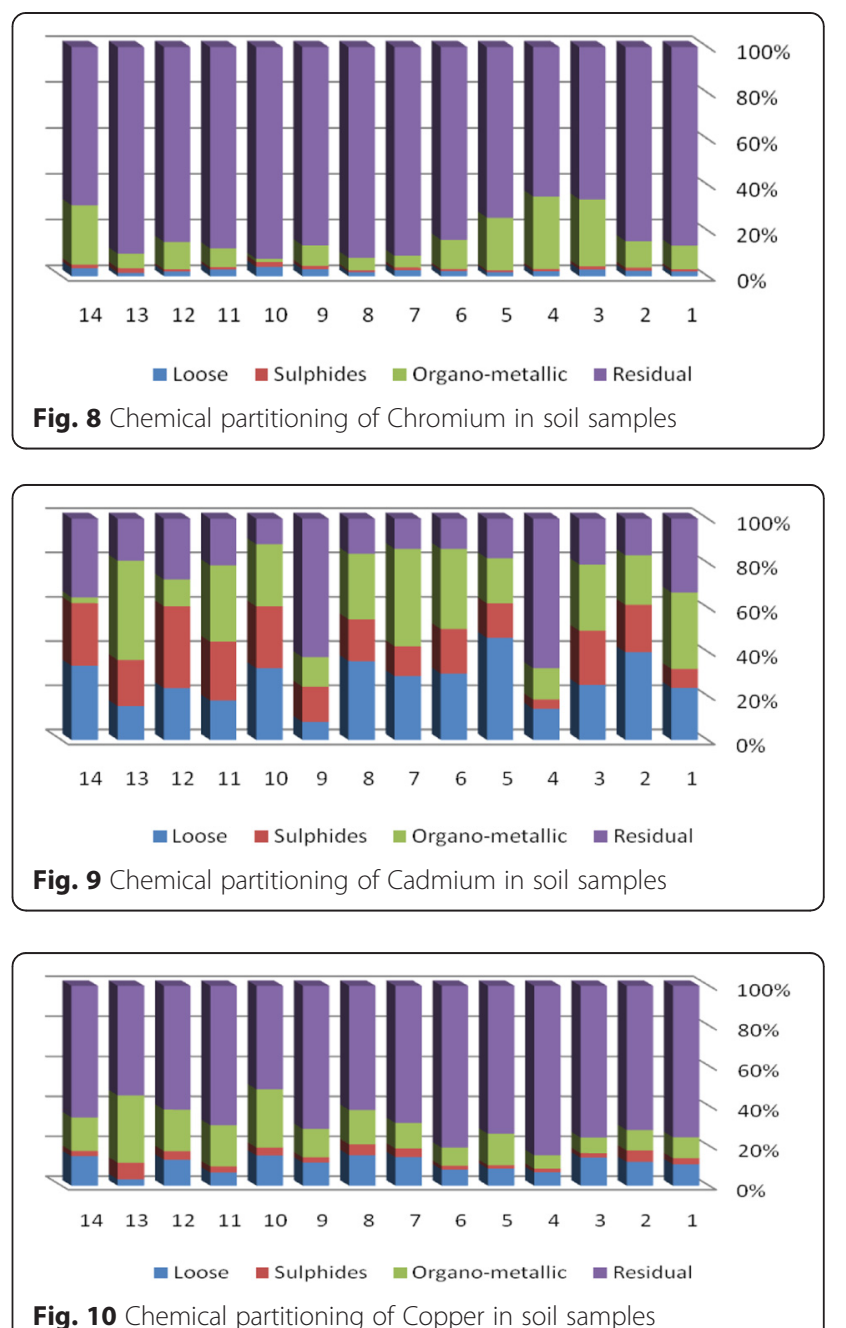

Fig. 10 Chemical partitioning of Copper in soil samples

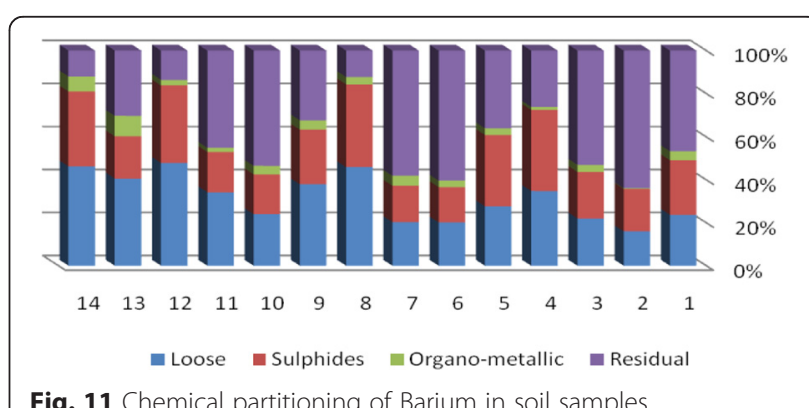

Fig. 11 Chemical partitioning of Barium in soil samples

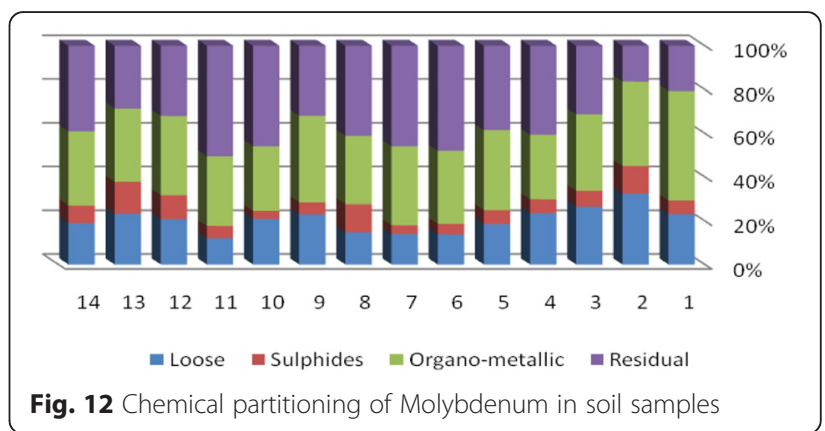



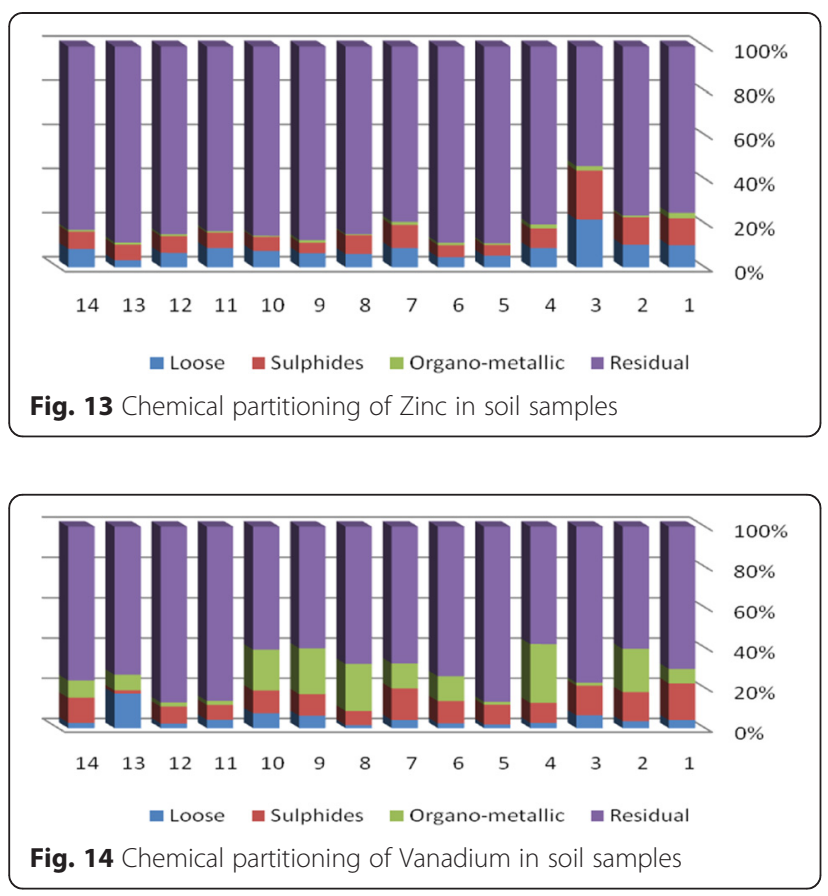

Chronic daily intakes of different metals within the framework of defined scenarios are compared with respective chronic oral reference doses to assess the existent hazards. Details for exposure assessment and hazard analysis are fully described within the literature [20].

\section{Results}

Total concentration of mentioned metals in soil samples collected from the vicinity of Mubarakeh steel complex are shown in Table 4.

In comparison with average shale values, all metals show lower concentrations except for Cd. Average cadmium concentration within the study area is more than five times higher than that of shale which indicate an intensified enrichment. On the other hand, although the concentration of other metals is lower than that of shale, such condition should not be considered as a low-risk and safe one. Potential mobility of existing metallic concentration in soil should be regarded before any judgment.

The results of chemical partitioning for metals are demonstrated in Figs. 3, 4, 5, 6, 7, 8, 9, 10, 11, 12, 13 and 14.

The percent of metals concentration bound to different phases are grouped in a descending order as follows:

Loose ions :

$\mathrm{Ba}(31.53)>\mathrm{As}(29.17)>\mathrm{Cd}(26.73)>\mathrm{Pb}(25.82)>\mathrm{Mo}$ $(20.37)>\mathrm{Co} \quad(19.21)>\mathrm{Mn} \quad(12.23)>\mathrm{Cu} \quad(10.98)>\mathrm{Zn}$ $(8.27)>\mathrm{V}(4.68)>\mathrm{Ni}(3.53)>\mathrm{Cr}(2.48)$

Sulfide ions :

$\mathrm{Mn}(36.97)>\mathrm{Co}(30.51)>\mathrm{Ba}(25.88)>\mathrm{Cd}(20.26)>\mathrm{Pb}$ $(19.22)>$ As $(17.48)>\mathrm{V}(10.92)>\mathrm{Zn}(8.96)>\mathrm{Mo}(7.74)>$ $\mathrm{Cu}(3.67)>\mathrm{Ni}(3.36)>\mathrm{Cr}(1.25)$

Organic ions :

Table 5 Percentile of anthropogenic portion in bulk metallic concentration of soil samples

\begin{tabular}{|c|c|c|c|c|c|c|c|c|c|c|c|c|c|c|}
\hline \multirow[t]{2}{*}{ Station } & \multicolumn{14}{|c|}{ Anthropogenic portion of metallic pollution (\%) } \\
\hline & Al & Co & $\mathrm{Mn}$ & As & $\mathrm{Ni}$ & $\mathrm{Pb}$ & $\mathrm{Cr}$ & $\mathrm{Cd}$ & $\mathrm{Cu}$ & $\mathrm{Ba}$ & $\mathrm{Fe}$ & Mo & $\mathrm{Zn}$ & V \\
\hline 1 & 11.54 & 72.31 & 34.58 & 66.14 & 11.80 & 74.41 & 12.01 & 59.90 & 21.86 & 47.99 & 0.78 & 71.34 & 22.27 & 26.47 \\
\hline 2 & 17.93 & 79.47 & 78.68 & 67.46 & 15.31 & 63.25 & 13.78 & 75.13 & 25.09 & 32.48 & 0.77 & 75.19 & 21.15 & 35.46 \\
\hline 3 & 5.74 & 64.45 & 58.75 & 70.40 & 22.32 & 72.17 & 30.21 & 71.34 & 21.77 & 42.31 & 2.29 & 61.82 & 41.24 & 20.26 \\
\hline 4 & 11.87 & 51.15 & 62.07 & 35.99 & 13.22 & 18.23 & 31.37 & 29.26 & 13.76 & 66.41 & 0.40 & 53.43 & 17.48 & 37.59 \\
\hline 5 & 4.12 & 66.29 & 34.02 & 33.28 & 7.82 & 29.40 & 22.99 & 73.88 & 23.44 & 57.60 & 0.21 & 55.32 & 9.83 & 11.79 \\
\hline 6 & 5.04 & 54.36 & 53.67 & 50.04 & 5.26 & 28.05 & 14.31 & 77.69 & 17.19 & 35.70 & 0.32 & 46.77 & 10.00 & 23.14 \\
\hline 7 & 5.44 & 75.91 & 43.47 & 47.32 & 8.14 & 39.16 & 8.00 & 77.62 & 28.30 & 37.74 & 0.73 & 48.67 & 18.58 & 28.94 \\
\hline 8 & 7.75 & 74.68 & 42.77 & 33.19 & 13.19 & 33.31 & 7.20 & 75.75 & 34.16 & 78.91 & 0.47 & 53.02 & 13.56 & 28.67 \\
\hline 9 & 7.68 & 56.89 & 53.93 & 52.16 & 10.37 & 58.51 & 12.13 & 33.73 & 25.59 & 60.73 & 0.32 & 61.23 & 11.07 & 35.70 \\
\hline 10 & 3.64 & 71.54 & 40.39 & 55.79 & 8.89 & 58.11 & 6.77 & 79.60 & 43.46 & 41.90 & 0.75 & 48.65 & 12.85 & 35.08 \\
\hline 11 & 2.03 & 71.01 & 33.00 & 45.29 & 6.24 & 36.63 & 10.91 & 71.00 & 27.29 & 49.42 & 0.33 & 44.50 & 14.76 & 12.15 \\
\hline 12 & 6.64 & 76.99 & 41.71 & 40.87 & 7.80 & 63.31 & 13.47 & 65.22 & 34.30 & 77.72 & 0.71 & 61.16 & 13.48 & 11.47 \\
\hline 13 & 9.94 & 77.84 & 33.28 & 39.72 & 6.18 & 38.57 & 8.84 & 72.83 & 40.69 & 62.64 & 0.19 & 64.24 & 10.13 & 23.88 \\
\hline 14 & 3.04 & 64.97 & 49.61 & 30.54 & 9.44 & 48.62 & 27.87 & 58.02 & 30.79 & 79.14 & 0.51 & 54.80 & 15.25 & 21.32 \\
\hline Max & 17.93 & 79.47 & 78.68 & 70.40 & 22.32 & 74.41 & 31.37 & 79.60 & 43.46 & 79.14 & 2.29 & 75.19 & 41.24 & 37.59 \\
\hline Min & 2.03 & 51.15 & 33.00 & 30.54 & 5.26 & 18.23 & 6.77 & 29.26 & 13.76 & 32.48 & 0.19 & 44.50 & 9.83 & 11.47 \\
\hline SD & 4.30 & 9.08 & 13.23 & 13.31 & 4.54 & 17.75 & 8.66 & 15.91 & 8.40 & 16.38 & 0.52 & 9.13 & 8.16 & 9.11 \\
\hline Mean & 7.31 & 68.42 & 47.14 & 47.73 & 10.43 & 47.27 & 15.70 & 65.78 & 27.69 & 55.05 & 0.63 & 57.15 & 16.55 & 25.14 \\
\hline
\end{tabular}


Table 6 Mean values of various pollution indices in soil samples

\begin{tabular}{|c|c|c|c|c|c|c|c|c|c|c|c|c|c|c|}
\hline Index & $\mathrm{Al}$ & Co & $\mathrm{Mn}$ & As & $\mathrm{Ni}$ & $\mathrm{Pb}$ & $\mathrm{Cr}$ & $\mathrm{Cd}$ & $\mathrm{Cu}$ & $\mathrm{Ba}$ & $\mathrm{Fe}$ & Mo & $\mathrm{Zn}$ & V \\
\hline Igeo & -6.75 & -3.50 & -1.54 & -1.28 & -0.75 & -2.00 & -0.36 & 1.94 & -0.57 & -3.10 & -2.14 & -3.04 & -1.19 & -3.10 \\
\hline Ipoll & 0.11 & 1.72 & 0.97 & 0.99 & 0.16 & 1.01 & 0.25 & 1.66 & 0.48 & 1.26 & 0.01 & 1.26 & 0.27 & 0.43 \\
\hline$E F$ & 0.04 & 0.44 & 1.45 & 17.05 & 2.38 & 2.03 & 1.42 & 48.67 & 2.60 & 0.64 & 1.00 & 1.04 & 3.31 & 0.45 \\
\hline
\end{tabular}

Mo $(35.39)>\mathrm{Co}(26.30)>\mathrm{Cd}(26.10)>\mathrm{Cu}(16.12)>\mathrm{Cr}$ $(13.72)>\mathrm{V}(12.33)>\mathrm{Pb}(7.48)>\mathrm{As}(6.38)>\mathrm{Ni}(4.65)>\mathrm{Ba}$ (3.75) $>\mathrm{Mn}(3.18)>\mathrm{Zn}(1.15)$

Residual ions :

$\mathrm{Ni} \quad(88.41)>\mathrm{Cr} \quad(82.55)>\mathrm{Zn} \quad(81.61)>\mathrm{V} \quad(72.07)>\mathrm{Cu}$

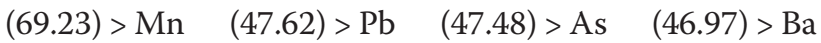
$(38.84)>\mathrm{Mo}(36.50)>\mathrm{Cd}(26.91) \mathrm{Co}(23.98)$
In order to have an overall estimate of metals mobility potential, the results of the sum of loose, sulfide and organic ions are grouped as the anthropogenic portion of metals are shown in Table 5 .

As was explained in methodology, IPOLL, Bc, and Lp refers to pollution intensity, bulk concentration, and lithogenous portion, respectively. Average indices

Table 7 concentration of metals in different rice samples from the study area ( $\mathrm{mg} / \mathrm{kg}$ dry weight)

\begin{tabular}{|c|c|c|c|c|c|c|c|c|c|c|c|c|c|c|}
\hline ID & $\mathrm{Al}$ & Co & $\mathrm{Mn}$ & As & $\mathrm{Ni}$ & $\mathrm{Pb}$ & $\mathrm{Cr}$ & $\mathrm{Cd}$ & $\mathrm{Cu}$ & $\mathrm{Ba}$ & $\mathrm{Fe}$ & Mo & $\mathrm{Zn}$ & V \\
\hline Rice 1 & 463.15 & 0.47 & 16.86 & 1.79 & 6.61 & 1.04 & 10.41 & 0.29 & 3.88 & 4.43 & 9.74 & 2.64 & 28.72 & 2.46 \\
\hline Rice 2 & 214.05 & 0.44 & 15.91 & 0.62 & 6.63 & 0.86 & 8.46 & 0.32 & 6.06 & 4.24 & 16.37 & 1.61 & 32.32 & 3.35 \\
\hline Rice 3 & 211.00 & 0.37 & 14.81 & 1.24 & 5.03 & 1.22 & 7.16 & 0.40 & 4.01 & 4.95 & 8.89 & 1.77 & 22.84 & 3.01 \\
\hline Rice 4 & 227.10 & 0.44 & 15.42 & 1.14 & 9.94 & 1.51 & 9.25 & 0.34 & 4.57 & 4.52 & 9.63 & 0.85 & 31.79 & 3.96 \\
\hline Rice 5 & 480.00 & 0.51 & 16.32 & 1.26 & 7.17 & 1.33 & 9.81 & 0.29 & 5.02 & 5.42 & 10.31 & 1.74 & 29.79 & 3.87 \\
\hline Rice 6 & 250.25 & 0.49 & 18.64 & 1.57 & 6.07 & 0.64 & 9.03 & 0.35 & 5.70 & 4.13 & 8.26 & 1.52 & 31.77 & 2.99 \\
\hline Rice 7 & 528.10 & 0.47 & 13.11 & 1.52 & 6.83 & 3.34 & 11.83 & 0.35 & 3.80 & 4.71 & 21.44 & 1.59 & 35.45 & 3.27 \\
\hline Rice 8 & 572.25 & 0.42 & 22.92 & 1.47 & 5.93 & 0.42 & 9.91 & 0.26 & 8.21 & 5.58 & 31.63 & 1.89 & 32.93 & 6.46 \\
\hline Mean & 368.24 & 0.45 & 16.75 & 1.33 & 6.78 & 1.30 & 9.48 & 0.33 & 5.16 & 4.75 & 14.53 & 1.70 & 30.70 & 3.67 \\
\hline St.Dev & 156.30 & 0.05 & 2.96 & 0.35 & 1.44 & 0.90 & 1.38 & 0.05 & 1.49 & 0.53 & 8.28 & 0.49 & 3.76 & 1.23 \\
\hline
\end{tabular}

Table 8 concentration of metals in different wheat samples from the study area ( $\mathrm{mg} / \mathrm{kg}$ dry weight)

\begin{tabular}{|c|c|c|c|c|c|c|c|c|c|c|c|c|c|c|}
\hline ID & $\mathrm{Al}$ & $\mathrm{Co}$ & $\mathrm{Mn}$ & As & $\mathrm{Ni}$ & $\mathrm{Pb}$ & $\mathrm{Cr}$ & $\mathrm{Cd}$ & $\mathrm{Cu}$ & $\mathrm{Ba}$ & $\mathrm{Fe}$ & Mo & $\mathrm{Zn}$ & V \\
\hline Wheat 1 & 294.85 & 0.46 & 52.17 & 1.11 & 5.89 & 1.23 & 8.55 & 1.26 & 9.23 & 5.26 & 11.29 & 1.93 & 38.55 & 6.61 \\
\hline Wheat 2 & 226.75 & 0.44 & 14.84 & 1.00 & 6.39 & 0.34 & 8.09 & 0.25 & 7.80 & 6.12 & 16.08 & 1.42 & 30.96 & 6.86 \\
\hline Wheat 3 & 329.35 & 0.53 & 53.54 & 1.95 & 6.87 & 1.09 & 9.37 & 0.25 & 10.67 & 7.34 & 13.81 & 1.81 & 19.85 & 8.91 \\
\hline Wheat 4 & 394.35 & 0.44 & 48.11 & 0.94 & 4.86 & 0.91 & 8.33 & 0.33 & 7.79 & 5.46 & 12.76 & 1.76 & 49.51 & 6.67 \\
\hline Wheat 5 & 493.70 & 0.46 & 53.86 & 3.11 & 6.77 & 1.07 & 10.42 & 1.16 & 7.90 & 8.00 & 14.38 & 1.55 & 28.56 & 7.29 \\
\hline Wheat 6 & 623.70 & 0.38 & 26.92 & 1.26 & 5.79 & 1.02 & 10.12 & 0.33 & 6.08 & 5.25 & 105.74 & 1.92 & 27.49 & 4.17 \\
\hline Wheat 7 & 344.15 & 0.47 & 61.94 & 1.13 & 6.92 & 0.49 & 9.80 & 0.27 & 11.38 & 7.40 & 17.49 & 1.86 & 49.32 & 7.92 \\
\hline Wheat 8 & 173.45 & 0.49 & 62.96 & 1.79 & 6.10 & 0.83 & 7.17 & 0.28 & 10.36 & 7.12 & 33.50 & 1.61 & 39.40 & 8.91 \\
\hline Wheat 9 & 148.70 & 0.54 & 70.96 & 1.98 & 10.18 & 1.92 & 7.51 & 0.38 & 11.50 & 7.87 & 18.96 & 1.39 & 41.68 & 8.84 \\
\hline Wheat 10 & 480.65 & 0.45 & 43.56 & 0.51 & 5.36 & 0.41 & 8.99 & 0.25 & 6.41 & 9.11 & 24.76 & 1.69 & 26.33 & 6.71 \\
\hline Wheat 11 & 230.25 & 0.71 & 83.25 & 2.29 & 7.48 & 1.23 & 7.76 & 0.25 & 12.81 & 10.22 & 27.59 & 1.47 & 54.04 & 11.05 \\
\hline Wheat 12 & 82.10 & 0.47 & 46.19 & 2.45 & 7.72 & 2.80 & 6.72 & 0.30 & 13.13 & 6.28 & 14.94 & 1.38 & 53.83 & 6.84 \\
\hline Wheat 13 & 185.30 & 0.48 & 63.82 & 1.94 & 7.35 & 1.19 & 10.22 & 0.29 & 8.42 & 12.26 & 10.21 & 1.86 & 46.50 & 8.21 \\
\hline Wheat 14 & 398.95 & 0.47 & 32.56 & 1.34 & 6.46 & 0.69 & 9.58 & 0.25 & 6.32 & 6.95 & 8.36 & 1.72 & 27.70 & 4.48 \\
\hline Wheat 15 & 499.10 & 0.43 & 54.17 & 1.15 & 5.87 & 0.43 & 9.05 & 0.26 & 8.14 & 5.13 & 20.83 & 2.02 & 41.91 & 8.11 \\
\hline Mean & 327.02 & 0.48 & 51.26 & 1.60 & 6.67 & 1.04 & 8.78 & 0.41 & 9.20 & 7.32 & 23.38 & 1.69 & 38.37 & 7.44 \\
\hline St.Dev & 154.67 & 0.07 & 17.33 & 0.69 & 1.26 & 0.64 & 1.16 & 0.33 & 2.32 & 2.00 & 23.79 & 0.21 & 10.99 & 1.74 \\
\hline
\end{tabular}


Table 9 Chronic daily intakes of metals through different scenarios due to rice consumption

\begin{tabular}{|c|c|c|c|c|c|c|c|c|}
\hline ID & $\begin{array}{l}\text { Mean concentration } \\
\mathrm{ppb}\end{array}$ & $\begin{array}{l}\text { Scenario } 1 \text { mg/ } \\
\text { kg-day }\end{array}$ & $\begin{array}{l}\text { Scenario } 2 \text { mg/ } \\
\text { kg-day }\end{array}$ & $\begin{array}{l}\text { Scenario } 3 \text { mg/ } \\
\text { kg-day }\end{array}$ & $\begin{array}{l}\text { Scenario } 4 \text { mg/ } \\
\text { kg-day }\end{array}$ & $\begin{array}{l}\text { Scenario } 5 \text { mg/ } \\
\text { kg-day }\end{array}$ & $\begin{array}{l}\text { Scenario } 6 \text { mg/ } \\
\text { kg-day }\end{array}$ & $\begin{array}{l}\text { Oral RfD mg/ } \\
\text { kg-day }\end{array}$ \\
\hline $\mathrm{Al}$ & 368.24 & $5.26 \mathrm{E}-01$ & $1.32 \mathrm{E}+00$ & $2.10 E+00$ & $2.45 \mathrm{E}+00$ & $6.14 \mathrm{E}+00$ & $9.82 \mathrm{E}+00$ & $1.00 E+00$ \\
\hline Co & 0.45 & $6.43 \mathrm{E}-04$ & $1.61 \mathrm{E}-03$ & $2.57 \mathrm{E}-03$ & $3.00 \mathrm{E}-03$ & $7.50 \mathrm{E}-03$ & $1.20 \mathrm{E}-02$ & $3.00 \mathrm{E}-04$ \\
\hline $\mathrm{Mn}$ & 16.75 & $2.39 \mathrm{E}-02$ & $5.98 \mathrm{E}-02$ & $9.57 \mathrm{E}-02$ & $1.12 \mathrm{E}-01$ & 2.79E-01 & 4.47E-01 & $1.40 \mathrm{E}-01$ \\
\hline As & 1.33 & 1.90E-03 & 4.75E-03 & 7.60E-03 & 8.87E-03 & $2.22 \mathrm{E}-02$ & 3.55E-02 & $3.00 \mathrm{E}-04$ \\
\hline $\mathrm{Ni}$ & 6.78 & 9.69E-03 & $2.42 \mathrm{E}-02$ & 3.87E-02 & 4.52E-02 & 1.13E-01 & 1.81E-01 & $2.00 \mathrm{E}-02$ \\
\hline $\mathrm{Cr}$ & 9.48 & 1.35E-02 & 3.39E-02 & $5.42 \mathrm{E}-02$ & $6.32 \mathrm{E}-02$ & $1.58 \mathrm{E}-01$ & $2.53 \mathrm{E}-01$ & $3.00 \mathrm{E}-03$ \\
\hline $\mathrm{Cd}$ & 0.33 & 4.71E-04 & $1.18 \mathrm{E}-03$ & $1.89 \mathrm{E}-03$ & $2.20 \mathrm{E}-03$ & $5.50 \mathrm{E}-03$ & 8.80E-03 & $1.00 \mathrm{E}-03$ \\
\hline $\mathrm{Cu}$ & 5.16 & 7.37E-03 & $1.84 \mathrm{E}-02$ & $2.95 \mathrm{E}-02$ & 3.44E-02 & $8.60 \mathrm{E}-02$ & 1.38E-01 & $4.00 \mathrm{E}-02$ \\
\hline $\mathrm{Ba}$ & 4.75 & $6.79 \mathrm{E}-03$ & $1.70 \mathrm{E}-02$ & 2.71E-02 & $3.17 \mathrm{E}-02$ & 7.92E-02 & $1.27 \mathrm{E}-01$ & $2.00 \mathrm{E}-01$ \\
\hline $\mathrm{Fe}$ & 14.53 & $2.08 \mathrm{E}-02$ & 5.19E-02 & 8.30E-02 & 9.69E-02 & $2.42 \mathrm{E}-01$ & 3.87E-01 & 7.00E-01 \\
\hline Mo & 1.7 & $2.43 \mathrm{E}-03$ & 6.07E-03 & $9.71 \mathrm{E}-03$ & $1.13 \mathrm{E}-02$ & $2.83 \mathrm{E}-02$ & 4.53E-02 & $5.00 \mathrm{E}-03$ \\
\hline $\mathrm{Zn}$ & 30.7 & 4.39E-02 & 1.10E-01 & $1.75 \mathrm{E}-01$ & 2.05E-01 & $5.12 \mathrm{E}-01$ & 8.19E-01 & $3.00 \mathrm{E}-01$ \\
\hline V & 3.67 & $5.24 \mathrm{E}-03$ & $1.31 \mathrm{E}-02$ & $2.10 \mathrm{E}-02$ & $2.45 \mathrm{E}-02$ & $6.12 \mathrm{E}-02$ & $9.79 \mathrm{E}-02$ & $7.00 \mathrm{E}-05$ \\
\hline
\end{tabular}

values for each metal in different stations are shown in Table 6.

The result of metal concentration in agricultural crops (wheat and rice), are shown in Tables 7 and 8.

According to defined scenarios in part of material and methods, respective chronic oral reference doses to assess the existent hazards are shown in Tables 9 and 10. Details for exposure assessment and hazard analysis are fully described within the literature [20].

As it is seen except for $\mathrm{Fe}, \mathrm{Ba}, \mathrm{Cu}$ and $\mathrm{Zn}$, all metals intakes in different scenarios are considered to be hazardous while their CDI values are much more than the respective oral reference doses.

\section{Discussion}

Evaluating the metallic pollution of soil and the potential mobility of metal species in the vicinity of Mubarakeh steel complex is considered in this study. Fourteen soil samples were collected and analyzed in summer 2012.

Bioavailability and health risks linked to toxic metals are tightly related to the mobility of metal species. aqueous organisms and plants are more vulnerable towards free ions and labile complexes [3, 21-23]. Accordingly, it may be concluded from the chemical partitioning data that there are significant differences in the distribution of the metals studied. Cobalt, $\mathrm{Cd}, \mathrm{As}, \mathrm{Mo}$ and $\mathrm{Pb}$ are introduced as the most risky metals since they present the

Table 10 Chronic daily intakes of metals through different scenarios due to wheat consumption

\begin{tabular}{|c|c|c|c|c|c|c|c|c|}
\hline ID & Mean concentration ppb & $\begin{array}{l}\text { Scenario } 1 \\
\text { mg/kg-day }\end{array}$ & $\begin{array}{l}\text { Scenario } 2 \\
\text { mg/kg-day }\end{array}$ & $\begin{array}{l}\text { Scenario } 3 \\
\text { mg/kg-day }\end{array}$ & $\begin{array}{l}\text { Scenario } 4 \\
\text { mg/kg-day }\end{array}$ & $\begin{array}{l}\text { Scenario } 5 \\
\text { mg/kg-day }\end{array}$ & $\begin{array}{l}\text { Scenario } 6 \\
\text { mg/kg-day }\end{array}$ & $\begin{array}{l}\text { Oral RfD } \\
\mathrm{mg} / \mathrm{kg} \text {-day }\end{array}$ \\
\hline $\mathrm{Al}$ & 327.02 & 7.01E-01 & $1.40 E+00$ & $2.10 E+00$ & $3.27 E+00$ & $6.54 E+00$ & $9.81 E+00$ & $1.00 E+00$ \\
\hline Co & 0.48 & 1.03E-03 & 2.06E-03 & 3.09E-03 & 4.80E-03 & 9.60E-03 & $1.44 \mathrm{E}-02$ & $3.00 \mathrm{E}-04$ \\
\hline $\mathrm{Mn}$ & 51.26 & $1.10 \mathrm{E}-01$ & $2.20 \mathrm{E}-01$ & 3.30E-01 & 5.13E-01 & $1.03 E+00$ & $1.54 \mathrm{E}+00$ & 1.40E-01 \\
\hline As & 1.6 & $3.43 \mathrm{E}-03$ & $6.86 \mathrm{E}-03$ & $1.03 \mathrm{E}-02$ & $1.60 \mathrm{E}-02$ & $3.20 \mathrm{E}-02$ & 4.80E-02 & $3.00 E-04$ \\
\hline $\mathrm{Ni}$ & 6.67 & $1.43 \mathrm{E}-02$ & $2.86 \mathrm{E}-02$ & 4.29E-02 & 6.67E-02 & $1.33 \mathrm{E}-01$ & 2.00E-01 & 2.00E-02 \\
\hline $\mathrm{Cr}$ & 8.78 & 1.88E-02 & $3.76 \mathrm{E}-02$ & 5.64E-02 & 8.78E-02 & 1.76E-01 & $2.63 \mathrm{E}-01$ & 3.00E-03 \\
\hline $\mathrm{Cd}$ & 0.41 & 8.79E-04 & $1.76 \mathrm{E}-03$ & 2.64E-03 & 4.10E-03 & $8.20 \mathrm{E}-03$ & $1.23 \mathrm{E}-02$ & $1.00 \mathrm{E}-03$ \\
\hline $\mathrm{Cu}$ & 9.2 & 1.97E-02 & 3.94E-02 & $5.91 \mathrm{E}-02$ & $9.20 \mathrm{E}-02$ & 1.84E-01 & $2.76 \mathrm{E}-01$ & 4.00E-02 \\
\hline $\mathrm{Ba}$ & 7.32 & 1.57E-02 & $3.14 \mathrm{E}-02$ & 4.71E-02 & 7.32E-02 & $1.46 \mathrm{E}-01$ & $2.20 \mathrm{E}-01$ & 2.00E-01 \\
\hline $\mathrm{Fe}$ & 23.38 & $5.01 \mathrm{E}-02$ & $1.00 \mathrm{E}-01$ & $1.50 \mathrm{E}-01$ & $2.34 \mathrm{E}-01$ & 4.68E-01 & 7.01E-01 & 7.00E-01 \\
\hline Mo & 1.69 & $3.62 \mathrm{E}-03$ & 7.24E-03 & 1.09E-02 & 1.69E-02 & $3.38 \mathrm{E}-02$ & 5.07E-02 & $5.00 \mathrm{E}-03$ \\
\hline $\mathrm{Zn}$ & 38.37 & $8.22 \mathrm{E}-02$ & $1.64 \mathrm{E}-01$ & 2.47E-01 & 3.84E-01 & 7.67E-01 & $1.15 E+00$ & 3.00E-01 \\
\hline V & 7.44 & 1.59E-02 & 3.19E-02 & 4.78E-02 & 7.44E-02 & 1.49E-01 & $2.23 \mathrm{E}-01$ & 7.00E-05 \\
\hline
\end{tabular}


highest percentages in loose, sulfide and organic fractions and the lowest in residual form. Accordingly, exchange of mentioned metals may be expected between the soil and water column.

Sum of loose, sulfide and organic phases in bulk metallic concentration is considered as manipulated contamination. The higher the anthropogenic portion of a metal is, the higher the mobility risk would be occurred. Generally, the mean anthropogenic portions of the metals, as a percent of their bulk concentrations, obey the following pattern: $\mathrm{Co}>\mathrm{Cd}>\mathrm{Mo}>\mathrm{Ba}>\mathrm{As}>\mathrm{Pb}>$ $\mathrm{Mn}>\mathrm{Cu}>\mathrm{V}>\mathrm{Zn}>\mathrm{Cr}>\mathrm{Ni}$.

\section{Conclusions}

Considering index approach, Igeo deals with bulk concentration and is constructed upon the principle of comparison with shale values. As it is expected, only cadmium is detected to be in a moderately contaminated status regarding this index while other metals declare an unpolluted condition. Index of pollution pays more attention to mobility potential of metals and accordingly detects metals $\mathrm{Co}, \mathrm{Mn}, \mathrm{As}, \mathrm{Pb}, \mathrm{Cd}, \mathrm{Ba}$ and $\mathrm{Mo}$ to be in a moderately contaminated level. On the other hand, enrichment factor which compares the bulk concentrations with earth crust values declares all toxic metals except for $\mathrm{Co}, \mathrm{Ba}$ and $\mathrm{V}$ to be enriched.

Considering human health hazard assessment, all toxic metals intakes in different scenarios are considered to be hazardous. The status is much more severe for scenarios dealt with children eating more portions. It must be noted that current hazard levels are calculated as single exposures and for more realistic conditions cumulative exposures should be taken into consideration. In other words, a variety of ingredients (containing rice, wheat, water, etc.) exists in diurnal diet of residents within the study area and the overall hazard would exceed the above-mentioned levels.

\section{Competing interests}

The authors declare that they have no competing interests.

\section{Authors' contributions}

All authors read and approved the final manuscript.

\section{Acknowledgements}

Authors want to appreciate kind cooperation of Mubarakeh steel complex and department of environment of Isfahan Province.

\section{Author details}

'Department of Environmental Science, Graduate School of the Environment and Energy, Science and Research Branch, Islamic Azad University, Tehran, Iran. ${ }^{2}$ Graduate Faculty of Environment, University of Tehran, P.O. Box 14155-6135 Tehran, Iran.

Received: 3 July 2013 Accepted: 13 October 2015

Published online: 30 October 2015

\section{References}

1. Adriano DC, Wenzel WW, Vangronsveld J, Bolan NS. Role of assisted natural remediation in environmental cleanup. Geoderma. 2004;122:121-42.

2. Martínez-Sánchez MJ, Navarro MC, Pérez-Sirvent C, Marimón J, Vidal J, García-Lorenzo ML, et al. Assessment of the mobility of metals in a mining-impacted coastal area (Spain, Western Mediterranean). J Geochem Explor. 2008;96:171-82.

3. Nasrabadi T, Nabi Bidhendi GR, Karbassi AR, Mehrdadi N. Partitioning of metals in sediments of the Haraz River (Southern Caspian Sea basin). Environ Earth Sci. 2010;59:1111-7.

4. Weisz M, Polyak K, Hlavay J. Fractionation of elements in sediment samples collected in rivers and harbors at lake Balaton and its catchment area. Microchem J. 2000;67:207-17.

5. Yu K-C, Tsai L-J, Chen S-H, Ho S-T. Correlation analyses binding behavior of heavy metals with sediment matrices. Water Res. 2001;35(10):2417-28.

6. Tessier A, Campbell PGC, Bisson M. Sequential extraction procedure for the speciation of particulate trace metals. Anal Chem. 1979;51:844-51.

7. Kersten M, Förstner U. Chemical fractionation of heavy metals in anoxic estuarine and coastal sediments. Water Sci Technol. 1986;18:121-30.

8. Ure AM, Quevauviller PH, Muntau H, Griepink B. Speciation of heavy metals in soils and sediments. An account of the improvement and harmonization of extraction techniques undertaken under the auspices of the BCR of the Commission of the European Communities. Int J Environ Anal Chem. 1993:51:135-51.

9. Vidal J, Pérez-Sirvent C, Martínez-Sánchez MJ, Navarro MC. Origin and behaviour of heavy metals in agricultural Calcaric Fluvisols in semiarid conditions. Geoderma. 2004;121:257-70.

10. Karbassi AR, Monavari SM, Nabi Bidhendi GR, Nouri J, Nematpour K. Metal pollution assessment of sediment and water in the Shur River. Environ Monit Assess. 2008;147:107-16.

11. Chester R, Hughes RM. A chemical technique for the separation of ferromanganese minerals, carbonate minerals and adsorbed trace elements from pelagic Sediment. Chem Geol. 1967;2:249-62.

12. Allen SE, Grimshaw HM, Rowland AP. Chemical analysis. In: Moore PD, Chapman SB, editors. Methods in plant ecology. Oxford, London: Blackwell, Scientific Publication; 1986. p. 285-344.

13. Nasrabadi T, Nabi Bidhendi GR, Karbassi AR, Mehrdadi N. Evaluating the efficiency of sediment metal pollution indices in interpreting the pollution of Haraz River sediments, southern Caspian Sea basin. Environ Monit Assess. 2010;171(1-4):395-410.

14. Lopez-Sanchez JF, Rubio R, Samitier C, Rauret G. Trace metal partitioning in marine sediments and sludges deposited off the coast of Barcelona (Spain). Water Res. 1996;30(1):153-9.

15. Jones B, Turki A. Distribution and speciation of heavy metals in surficial sediments from the Tees estuary, northeast England. Mar Pollut Bull. 1997;34(10):768-79.

16. Datta DK, Subramanian V. Distribution and fractionation of heavy metals in the surface sediments of the Ganges-Brahmaputra-Meghna river system in the Bengal basin. Environ Geol. 1998;36(1-2):93-101.

17. Gonzalez-Macias C, Schifter I, Lluch-cota DB, Mendez-Rodriguez L, HernandezVazquez S. Distribution, enrichment and accumulation of heavy metals in coastal sediments of Salina Cruz Bay, Mexico. Environ Monit Assess. 2006;118:211-30.

18. Forstner U, Ahlf W, Calmano W, Kersten M. Sediment criteria developmentcontributions of environmental geochemistry to water quality management. In: D Helling, P Rothe, U Forstner, P. Stoffers editors. Sediments and environmental geochemistry. Berlin: Springer; 1990. pp 311-338.

19. Pekey H. Heavy metal pollution assessment in sediments of the Izmit Bay, Turkey. Environ Monit Assess. 2006;123:219-31.

20. Risk Assessment Information System (RAIS). USEPA (Electronic data base). 2009. http://rais.ornl.gov/.

21. Ibrahim Korfali S, Davies BE. Speciation of metals in sediment and water in a river underlain by limestone: role of carbonate species for purification capacity of rivers. Adv Environ Res. 2004;8:599-612.

22. Murakami M, Fujita M, Furumai H, Kasuga I, Kurisu F. Sorption behavior of heavy metal species by soakaway sediment receiving urban road runoff from residential and heavily trafficked areas. J Hazard Mater. 2009;164:707-12.

23. Degryse F, Smolders E, Merckx R. Labile Cd complexes increase Cd availability to plants. Environ Sci Technol. 2006;40:830-6. 